\title{
Studies of uranium carbide targets of a high density
}

\author{
V.N. Panteleev ${ }^{a, *}$, O. Alyakrinskiy ${ }^{b}$, M. Barbui ${ }^{b}$, A.E. Barzakh ${ }^{a}$, M. Dubois ${ }^{c}$, \\ C. Eleon ", S. Essabaa ", D.V. Fedorov", G. Gaubert ", A.M. Ionan ", V.S. Ivanov", \\ P. Jardin ${ }^{c}$, C. Lau ${ }^{d}$, R. Leroy ${ }^{c}$, G. Lhersonneau ${ }^{c}$, K.A. Mezilev ${ }^{\text {a', }}$

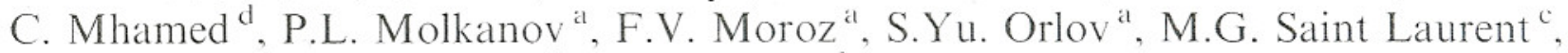 \\ L. Stroe $^{b}$, L.B. Tecchio ${ }^{b}$, M. Tonezzer ${ }^{b}$, A.C.C. Villari ${ }^{c}$, Yu.M. Volkov ${ }^{a}$ \\ "Petershurg Nuclear Physics Institute RAS. 188300 Guthina. Russia \\ ${ }^{b}$ Laboratori Nationali di Lergnaro, 3.5020) Legnaro, Padola. Iraly. \\ "GANIL. F-14076 Cian Cedex 5. France" \\ 'Institut de Physique Nucleaire, F-91406 Orsay Cedex, France
}

Available online 5 June 2008

\begin{abstract}
Production of Cs and Fr isotopes from uranium carbide targets of a high density has been investigated at IR IS (Investigation Radioactive Isotopes at Synchrocyclotron). Gatchina. The UC target material with a density of $12 \mathrm{~g} / \mathrm{cm}^{3}$ was prepared in a form of pellets. Two targets were tested on-line under the same temperature conditions: (a) a reference small target with a thickness of $4.5 \mathrm{~g} / \mathrm{cm}^{2}:$ (b) a heavier (so called intermediate) target with a thickness of $91 \mathrm{~g} / \mathrm{cm}^{2}$. Yields and release efficiencies of nuclides with half-lives from some minutes to some milliseconds produced by $1 \mathrm{GeV}$ protons in these targets are presented. It is remarkable that yields, even those of very short-lived isotopes such as ${ }^{214} \mathrm{Fr}\left(T_{1 / 2}=5 \mathrm{~ms}\right)$ and ${ }^{219} \mathrm{Fr}\left(T_{1 / 2}=20 \mathrm{~ms}\right)$. increase proportionally to the target thickness. A one month off-line heating test of the $91 \mathrm{~g} / \mathrm{cm}^{2}$ target at a temperature of $2000{ }^{\circ} \mathrm{C}$ has been carried out successfully. The yields and release efficiencies of Cs and Fr measured on-line before and after the heating test coincided within the limits of measurement errors, thereby demonstrating the conservation of the target unit parameters. Based on these very promising results. a heavier target with a mass about $0.7 \mathrm{~kg}$ is prepared presently at IRIS.
\end{abstract}

(C) 2008 Elsevier B.V. All rights reserved.

PACS: $25.40 . \mathrm{Sc}: 28.60 .+\mathrm{S}: 29.25 . \mathrm{Ni}: 29.25 . \mathrm{Rm}$

Kẹwords: Uranium carbide target: Ionising target: Massive target; Yield: Delay time

\section{Introduction}

This work is devoted to off-line and on-line investigations of uranium carbide target of a high density [1,2] to establish whether these targets are efficient for production of neutron-deficient and neutron-rich nuclei far from stability. The next generation ISOL facilities $[3,4]$, where neutron converters will be used for secondary neutron

Corresponding author. Tel: +78127136041 (reception). +7812 7146208 (office): fax: +078127136041 .

E-mail address; vnp(apnpi.spb.ru (V.N. Panteleev). production. require uranium carbide target having a big mass up to some kilograms. Presently there is no universal model for reliable calculations of the target release characteristics determined mainly by diffusion and effusion processes to estimate the release parameters of such a big target. So the prediction of the produced yields for a very massive target can give an error up to some order of magnitude. To investigate the influence of the target mass and volume increase on the target characteristics. the tests of the target with the "intermediate" mass of uranium carbide of about $100 \mathrm{~g}$ were carried out to compare its characteristics (yields and delay parameters) with those of the smaller 
reference target. The results presented here focus on the development of this intermediate mass UC target, namely long-term stability and comparison of the yields of $\mathrm{Cs}$ and $\mathrm{Fr}$ isotopes with those obtained with the reference target. The following step in the development of high density targets is the construction and testing of a target prototype with UC mass about $700 \mathrm{~g}$, which is $1 / 3$ of the current design of SPIRAL-2 [4].

\section{Target construction and experiment description}

A schematic view of the used target assemblies is presented in figs. 1 and 2. The ionizing target described in $[1,5]$ has been used as a reference small target (Fig. 1). This small target with high density pellets (HDP) had uranium density $=(11.5 \pm 0.5) \mathrm{g} / \mathrm{cm}^{3}$ and grain size about $20 \mu \mathrm{m}$. Dimensions were: thickness $=4.5 \mathrm{~g} / \mathrm{cm}^{2}$, mass $=4.4 \mathrm{~g}$, length $=3.9 \mathrm{~mm}$, diameter $=11.2 \mathrm{~mm}$. There were 2 pellets, each of $1.9 \pm 0.1 \mathrm{~mm}$ thickness. The first containment was a graphite tube with inner diameter $12 \mathrm{~mm}$ and wall thickness about $1 \mathrm{~mm}$. The external container in tungsten-tantalum was $57 \mathrm{~mm}$ long and $16 \mathrm{~mm}$ in diameter, Since we intended to compare yields of nuclides with low ionization potentials, namely the alkalis $\mathrm{Rb}, \mathrm{Cs}$ and Fr, the small ionizing target did not have an ionizer. As a matter of fact, ionization of these elements occurs on a hot inner surface of the tungsten-tantalum target container with a high efficiency. The application of the appropriate sign of potential of DC current heating the target container can direct the produced radioactive ions to the exit target hole towards extraction electrode accelerating by this way the effusion process.

An intermediate high density pellet target (Fig. 2) contained pellets of the same target material and dimensions as used in the reference target, placed in two graphite tubes with the inner diameter of $12 \mathrm{~mm}$. The target thickness was $91 \mathrm{~g} / \mathrm{cm}^{2}$, target mass was $90 \mathrm{~g}$. The external two tungsten-tantalum containers, each $70 \mathrm{~mm}$ length and $16 \mathrm{~mm}$ in diameter, were connected to a surface ionizer. The ionizer was a rhenium tube with inner diameter of $1.5 \mathrm{~mm}$. The targets were exposed to $1 \mathrm{GeV}$ proton beam of intensity of $0.05-0.07 \mu \mathrm{A}$. As in our experiments the beam spot dimension was considerably larger than the target cross-section and the distribution of the proton beam intensity across the target was uniform in both used geometries (parallel or perpendicular beam direction), the target thickness in both cases was calculated by the same way along the target axe. In both cases as well the proton beam intensity was measured through the cross-section of $11.2 \mathrm{~mm}$ in diameter.

The isotopes were selected by the field of the mass-separator magnet, collected on a tape and transported to appropriate detectors to be identified by characteristic $\alpha$-, $\beta$ - and $\gamma$-radiation. A more detailed description of the measurement procedure and the yield determination from the obtained data can be found in $[2,6]$.

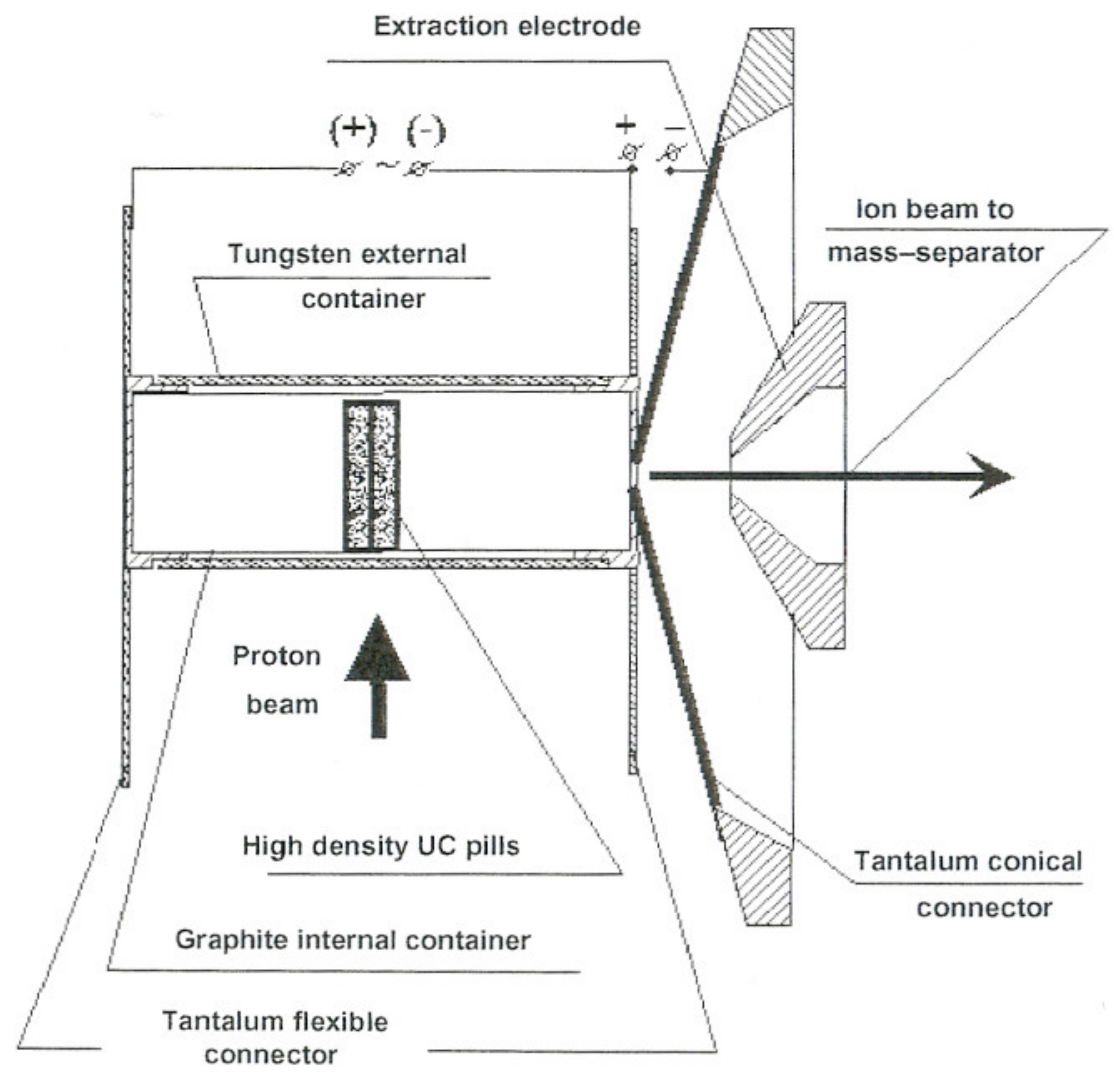

Fig. 1. Schematic view of a small target. 


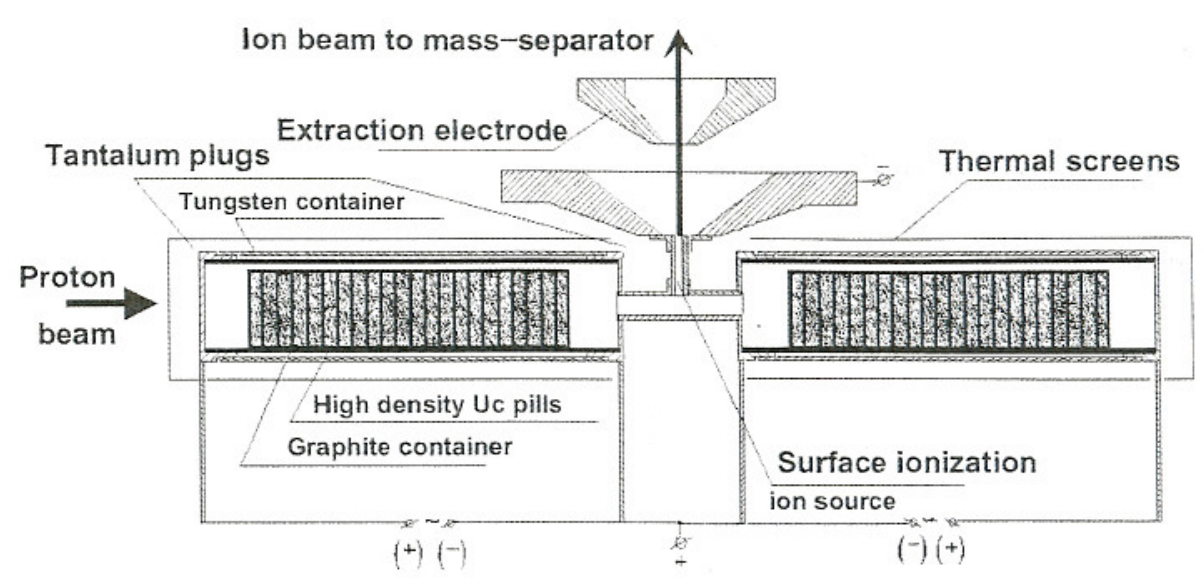

Fig. 2. Schematic view of an intermediate target.

\section{Experimental results and discussion}

For the targets to be compared the yields and release times have been measured in similar temperature conditions in the temperature interval of $1900-2100^{\circ} \mathrm{C}$. All the yields shown are normalized to a target thickness of $1 \mathrm{~g} / \mathrm{cm}^{2}$ and $0.1 \mu \mathrm{A}$ proton beam current. The so-called "normalized" yields of neutron-rich Cs isotopes from a small $\left(4.5 \mathrm{~g} / \mathrm{cm}^{2}\right)$ and intermediate $\left(91 \mathrm{~g} / \mathrm{cm}^{2}\right)$ HDP targets are presented in Fig. 3. The yield values of $\mathrm{Rb}, \mathrm{Cs}$ and $\mathrm{Fr}$ isotopes from a small $4.5 \mathrm{~g} / \mathrm{cm}^{2}$ target were measured in December 2005 [6]. In the table A 2.11 of [6] Rb and Cs yields measured only by $\gamma$-detector were given. In this paper the final evaluation of the data, taking into account also the $\beta$-measurements have been presented. The Fr yields obtained at $T=2190^{\circ} \mathrm{C}$ were processed later and therefore have not been included into the data of [6].

The trends with a mass number for a small and intermediate target - as well before and after one month heating are rather similar. The yields from $91 \mathrm{~g} / \mathrm{cm}^{2}$ target before and after the heating test are equal within the limits of measurement errors. This confirms that the properties of the

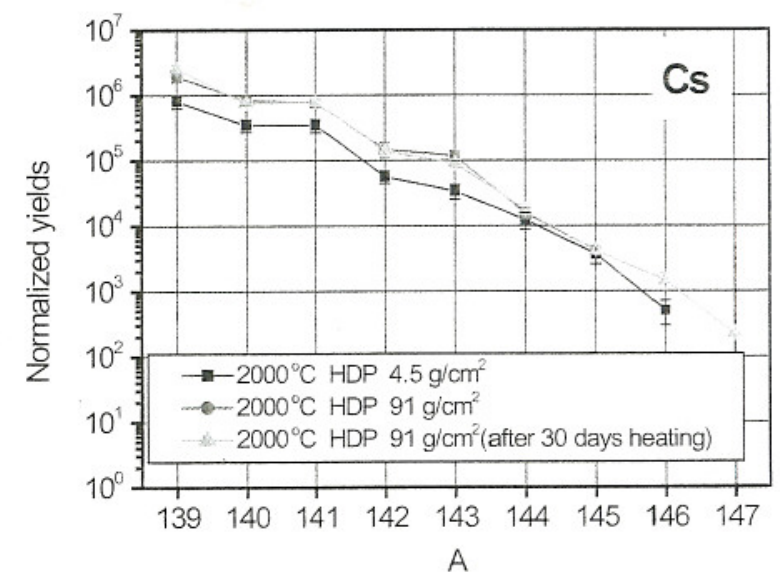

Fig. 3. Normalized yields of neutron-rich Cs isotopes from a small $(4.5 \mathrm{~g} /$ $\left.\mathrm{cm}^{2}\right)$ and intermediate $\left(91 \mathrm{~g} / \mathrm{cm}^{2}\right)$ HDP targets. target-ion source unit have not changed during the heating period.

Globally, the normalized yields from the intermediate target are about two times higher than those from the smaller reference target. This increase can be explained by the neutron-rich isotope yield enhancement due to higher amount of reactions produced by secondary neutrons in the heavier mass target.

The release curves of long-lived ${ }^{139} \mathrm{Cs}\left(T_{1 / 2}=9.27 \mathrm{~min}\right)$ measured at $T=2000{ }^{\circ} \mathrm{C}$ (not presented here) are close for all investigated targets. Similarly to the results obtained in our earlier works for high density UC targets [1,7] the measured release curves have a fast and slow component of about one and twenty minutes with errors up to $40 \%$. To define more precisely a long component of the release curve an isotope with a half-life considerably longer than expected delay time should be used. In Fig. 4 the release curve measured with ${ }^{132} \mathrm{Cs}$ ( $T_{1 / 2}=6.48$ days $)$ is presented. The longest component can be reliably measured in that case. It is about $4 \mathrm{~h}$. It seems possible that the longest component measured with ${ }^{139} \mathrm{Cs}$ is a mixture of the short and long components measured now. The release curve of long-lived ${ }^{132} \mathrm{Cs}$ was measured from the target with three pills of the same HDP target material of $6.5 \mathrm{~g} / \mathrm{cm}^{2}$ thickness.

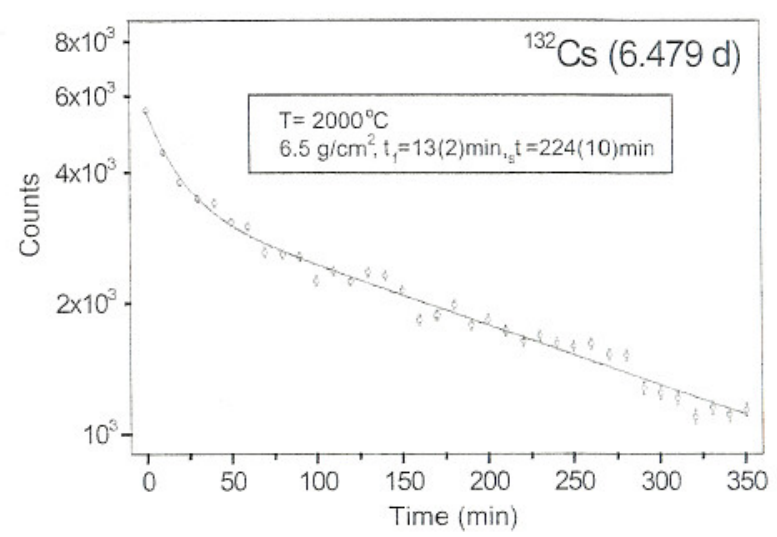

Fig. 4. Release curve measured with ${ }^{132} \mathrm{Cs}\left(T_{1 / 2}=6.48\right.$ days $)$. 


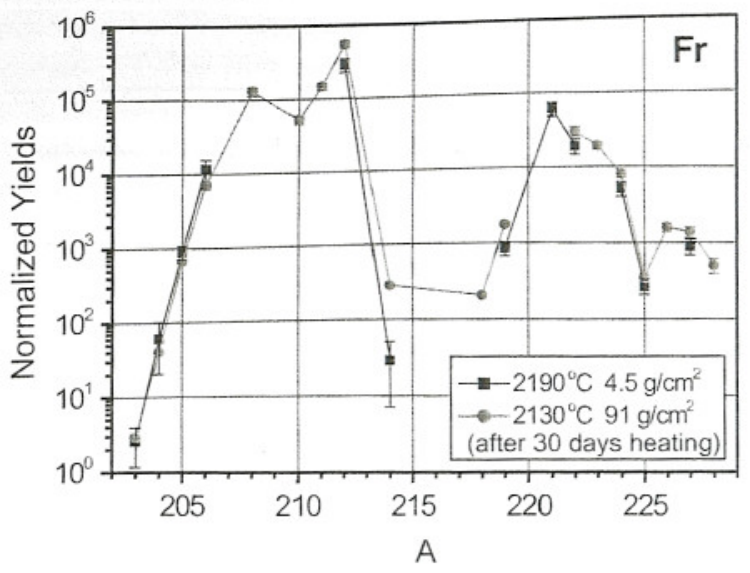

Fig. 5. Normalized yields of Fr isotopes from a small $\left(4.5 \mathrm{~g} / \mathrm{cm}^{2}\right)$ and intermediate $\left(91 \mathrm{~g} / \mathrm{cm}^{2}\right)$ HDP targets.

In Fig. 5 the normalized yields of $\mathrm{Fr}$ isotopes extracted from the $4.5 \mathrm{~g} / \mathrm{cm}^{2}$ and $91 \mathrm{~g} / \mathrm{cm}^{2}$ HDP targets are presented. Therefore, absolute yields increase proportionally to the ratio of the target thicknesses. To compare the release properties of the tested targets, the measured yields of Fr isotopes from a big target have been divided by the yields of the same isotopes from a small target and normalized to the unity at the ${ }^{222} \mathrm{Fr}$ (half-life $14.2 \mathrm{~min}$ ). Plot of these ratios, as a function of the isotope half-lives, is shown in Fig. 6. It shows that the ratio of normalized yields of Fr isotopes from a small and big target is close to 1 within the limits of measurement errors in a wide range of half-lives. The most remarkable result is that there are no loses of the yields of very short-lived $\mathrm{Fr}$ isotopes ${ }^{214} \mathrm{Fr}\left(T_{1 / 2}=\right.$ $5 \mathrm{~ms})$ and ${ }^{219} \mathrm{Fr}\left(T_{1 / 2}=20 \mathrm{~ms}\right)$, when the target mass was increased by twenty times. An unexpected high ratio for ${ }^{214} \mathrm{Fr}$ can be explained by a high measurement error of this isotope yield for a small reference target. In Fig. 7 the plot of the ratios of the yields of Cs isotopes from the intermediate and small target versus isotope half-lives is presented. The ratios have been normalized to the unity at the point of ${ }^{140} \mathrm{Cs}\left(T_{1 / 2}=1.05 \mathrm{~min}\right)$. As one can see, similar to the Fr

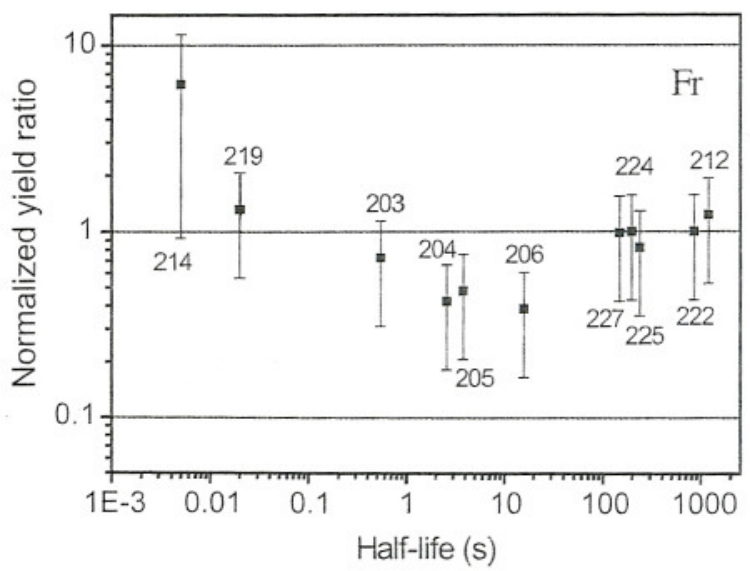

Fig. 6. Ratio of normalized yields of $\mathrm{Fr}$ isotopes from an intermediate $\left(91 \mathrm{~g} / \mathrm{cm}^{2}\right)$ HDP and a small $\left(4.5 \mathrm{~g} / \mathrm{cm}^{2}\right)$ targets.

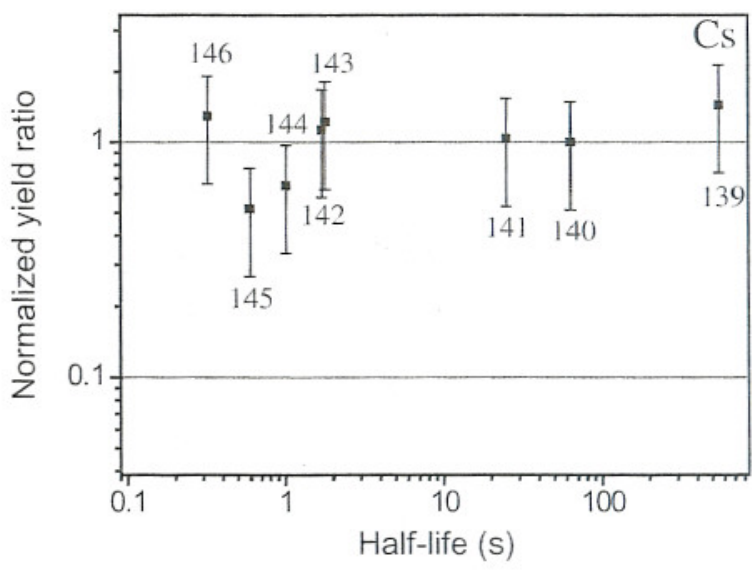

Fig. 7. Ratio of normalized yields of Cs isotopes from an intermediate $\left(91 \mathrm{~g} / \mathrm{cm}^{2}\right)$ HDP and a small $\left(4.5 \mathrm{~g} / \mathrm{cm}^{2}\right)$ targets.

isotope yields, there are no losses of the yields of Cs short-lived isotopes from the intermediate mass target. But situation with Cs isotopes is not so evident as for Fr. Some compensation of the yield losses can be due to the short-lived isotope yield enhancement by secondary neutrons in the heavier mass target. The question still remains about a lower overall efficiency of the HDP material comparing to HDR (high density rod) or LDP (low density pill) target materials [6]. Preliminary we can suggest that it is due to a very long component of the release (see Fig. 4). Additional on-line tests will be carried out to verify this suggestion.

The very encouraging results about alkali yields increase proportionally to the target mass have stimulated the construction of a new even bigger target with target material mass of about $0.7 \mathrm{~kg}$, which is presently tested off-line at the IRIS test bench. The new high density pellet target has the following characteristics: pellets of $25 \mathrm{~mm}$ in diameter and $2 \mathrm{~mm}$ thickness made of already used $5 \mu \mathrm{m}$ grain target material. The UC pellets are contained in two internal graphite tubes with the inner diameter of $26 \mathrm{~mm}$. The target thickness is about $160 \mathrm{~g} / \mathrm{cm}^{2}$. The second containment consists of two tungsten-tantalum containers connected to a tungsten tube of $1.5 \mathrm{~mm}$ inner diameter acting as a surface ionizer. A tungsten tube is used as a surface ionizer. Each of the external tungsten-tantalum containers is $100 \mathrm{~mm}$ in length and $30 \mathrm{~mm}$ in diameter.

\section{Conclusion}

The yields and release of on-line mass-separated $\mathrm{Rb}, \mathrm{Cs}$ and $\mathrm{Fr}$ isotopes (in this paper only $\mathrm{Cs}$ and $\mathrm{Fr}$ are discussed) produced by fission and spallation of ${ }^{238} \mathrm{U}$ induced by $1 \mathrm{GeV}$ protons have been studied for a high density UC pill targets with the target material thicknesses of $4.5 \mathrm{~g} / \mathrm{cm}^{2}$ and $91 \mathrm{~g} / \mathrm{cm}^{2}$ (intermediate mass target). The yields and release curves of $\mathrm{Cs}$ isotopes were measured from the intermediate target before and after 1 month heating test without beam and remained both unchanged. The yields 
of $\mathrm{Fr}$ and $\mathrm{Cs}$ isotopes measured for a small and intermediate target have shown the produced yield increase for tested target proportionally to the ratio of the target thicknesses even for very short-lived isotopes ${ }^{214} \mathrm{Fr}\left(T_{1 / 2}=5 \mathrm{~ms}\right)$. ${ }^{219} \mathrm{Fr}\left(T_{1 / 2}=20 \mathrm{~ms}\right)$ and ${ }^{146} \mathrm{Cs}\left(T_{1 / 2}=321 \mathrm{~ms}\right)$. It is a very encouraging result as it shows that the limit of the target mass increase has not been reached yet for production of short-lived alkalis. Consequently, the construction and off-line testing of a UC target with uranium mass of about $0.7 \mathrm{~kg}$ have been initiated.

A very long component of the release curve which of about 4 h have been observed using the 6.49 days ${ }^{132} \mathrm{Cs}$ isotope. The reason for such a slow release process of alkalis will be investigated in following experiments.

\section{Acknowledgements}

We acknowledge the financial support of the European Community under the FP6 "Research Infrastructure Action-Structuring the European Research Area" EURISOL DS Project Contract No. 515768 RIDS. The EC is not liable for the use that can be made of the information contained herein. For PNPI this work has been supported by ISTC Project \# 2965.

\section{References}

[1] V.N. Panteleev, A.E. Barzakh, D.V. Fedorov, A.M. Ionan, V.S. Ivanov, K.A. Mezilev, F.V. Moroz, S.Yu. Orlov, Yu.M. Volkov, A. Andrighetto, E. Del Piero, G. Lhersonneau, L. Stroe, V. Rizzi, L.B.
Tecchio, M. Dubois, G. Gaubert. P. Jardin. N. Lecesne, R. Leroy, J.Y. Pacquet, M.G. Saint Laurent. A.C.C. Villari. O. Bajeat. S. Essabaa, C. Lau, M. Menna. Development of uranium carbide targets for the online production of neutron-rich isotopes. Nucl. Instr. and Meth. B 240 (2005) 888

[2] A. Andrighetto, A.E. Barzakh, D.V. Fedorov, V.S. Ivanov, G. Lhersonneau, F.V. Moroz, S.Yu. Orlov, V.N. Panteleev, M.D. Seliverstov, I.M. Strachnov, L. Stroe, L.B. Tecchio, Yu.M. Volkov. X.F. Wang, Proton- and neutron-induced fission on uranium carbide target. Eur. Phys. J. A 19 (2004) 341.

[3] A. Bracco, A. Pisent (Eds.). SPES: Technical Design for an Advanced Exotic Ion Beam Facility at LNL. LNL-INFN (Rep) 181/02, 2002.

[4] A.C.C.Villari, in: Villari, The SPIRAL2 project at GANIL. AIP Conf. Proc. 704 (2004) 234.

[5] V.N. Panteleev, A.E. Barzakh, D.V. Fedorov. A.M. Ionan. K.A. Mezilev, F.V. Moroz, S. Yu. Orlov, Yu.M. Volkov, A. Andrighetto. G. Lhersonneau, V. Rizzi, L.B. Tecchio, M. Dubois. G. Gaubert, P. Jardin. N. Lecesne, R. Leroy, J.Y. Pacquet. M.G. Saint Laurent. A.C.C. Villari, O. Bajeat, S. Essabaa, C. Lau, M. Menna, Combined target-ion source unit for production of rare nuclides, Rev. Scientific Instrum. 77 (2006) 03A705.

[6] O. Alyakrinskiy, A. Andrighetto, O. Bajeat, A.E. Barzakh. M. Cheikh Mhamed, M. Dubois, C. Eléon, S. Essabaa. D.V. Fedorov, G. Gaubert, A.M. Ionan, V.S. Ivanov, P. Jardin. A. Lanchais, C. Lau, N. Lecesne, R. Leroy, M.P. Levchenko. G. Lhersonneau. K.A. Mezilev. F.V. Moroz, S.Yu. Orlov, J.Y. Pacquet, V.N. Panteleev, V. Rizzi, B. Roussière, M.-G. Saint-Laurent, L. Stroe, L.B. Tecchio, A.C.C. Villari, Yu.M. Volkov, Report on the R\&D of Uranium Carbide Targets by the PLOG Collaboration, EURISOL Design Study. task 4, 2007.

[7] A. Andrighetto, O. Bajeat, A.E. Barzakh, S. Essabaa, D.V. Fedorov, A.M. Ionan, V.S. Ivanov, R. Leroy. G. Lhersonneau. K.A. Mezilev, F.V. Moroz, S.Yu. Orlov, V.N. Panteleev, L. Stroe, L.B. Tecchio, A. Villari, Yu.M. Volkov, X.F. Wang. On-line production of Rb and Cs isotopes from uranium carbide targets, Eur. Phys. J. A 23 (2005) 257. 